\title{
Résolution de l'équation de diffusion par une méthode semi-implicite. Application au cas de la radiolyse de l'eau par des électrons
}

\author{
A. SAIFI*
}

(Manuscrit reçu le 14 juin 1994, révisé le 8 juin 1995, accepté le 14 septembre 1995)

RÉSUMÉ De par les hypothèses simplificatrices qui les caractérisent (distribution homogène des dépôts d'énergie, contribution des électrons de basses énergies négligée, données biologiques limitées, etc.), les évaluations dosimétriques effectuées à l'échelle macroscopique peuvent conduire à des erreurs considérables par surestimation ou sous-estimation de la dose délivrée. Une meilleure connaissance des débits de dose à l'échelle cellulaire passe par une estimation de la distribution spatiale des espèces radiolytiques. En effet, ce travail consiste à étudier les processus de diffusion et de réaction des particules apparues lors de la phase physico-chimique $\left(\mathrm{e}^{-}\right.$aq $, \mathrm{H}, \mathrm{OH}, \mathrm{H}^{+}$aq $\left., \mathrm{H}_{2}, \mathrm{H}_{2} \mathrm{O}_{2}\right)$. Pour ce faire, nous avons développé une technique de résolution de l'équation de diffusion. Celle-ci est, en fait, une équation aux dérivées partielles non linéaires à coefficients constants. Dans ce qui suit, nous décrivons les différentes étapes menant à la résolution de l'équation ainsi que les codes numériques établis à cet effet. Les principaux objectifs sont : 1) le calcul des concentrations et des rendements radiochimiques des espèces : $\mathrm{e}^{-}$aq $, \mathrm{H}, \mathrm{OH}, \mathrm{H}^{+}{ }_{\text {aq }}, \mathrm{H}_{2}, \mathrm{H}_{2} \mathrm{O}_{2}, \mathrm{OH}^{-}, \mathrm{O}_{2}, \mathrm{O}_{2}^{-}, \mathrm{HO}_{2}$, $\mathrm{HO}^{-}$; 2) la comparaison des rendements radiochimiques obtenus par application des méthodes explicite et semi-implicite pour des électrons incidents d'énergies $500 \mathrm{eV}$ et $10 \mathrm{keV}$. Les principales conclusions sont : l'inefficience des méthodes analytiques avant l'écoulement d'un temps de l'ordre de la microseconde ; la prise en compte d'un soluté quelconque n'est nécessaire qu'autour de la microseconde pour réduire les rendements radiochimiques des particules à l'origine du déséquilibre du milieu irradié (eau).

ABSTRACT Dosimetric evaluations usually carried out at a macroscopic scale by using simplified assumptions (homogeneous energy distribution, contribution of lowenergy electrons neglected, restricted biological data, ...) can lead to significant errors due to over-estimation or under-estimation of the delivered dose. Thus, an estimation of the spatial distribution of radiolytic species is needed in order to arrive at a better knowledge of the dose rate at the cellular level. The aim of this work is to study the processes of diffusion and reaction of particles $\left(\mathrm{e}^{-}{ }_{\mathrm{aq}}, \mathrm{H}\right.$, $\mathrm{OH}, \mathrm{H}^{+}{ }_{\text {aq }}, \mathrm{H}_{2}, \mathrm{H}_{2} \mathrm{O}_{2}$ during the physico-chemical step. A technique of resolution of the equation of diffusion has been therefore developed. The latter is in

\footnotetext{
* Institut d'électronique, Université de Constantine, Route de Aïn-El-Bey, 25000 Constantine, Algérie.
} 
fact an equation with non linear partial derivatives with constant coefficients. The different stages leading to the solution of this equation are described as well as the numerical codes established to this effect. The main objectives are : 1) the calculation of the concentrations and yields of species : $\mathrm{e}^{-}{ }_{\mathrm{aq}}, \mathrm{H}, \mathrm{OH}, \mathrm{H}^{+}{ }_{\mathrm{aq}}, \mathrm{H}_{2}$, $\left.\mathrm{H}_{2} \mathrm{O}_{2}, \mathrm{OH}^{-}, \mathrm{O}_{2}, \mathrm{O}_{2}^{-}, \mathrm{HO}_{2}, \mathrm{HO}_{2}^{-} ; 2\right)$ a comparison of the yields obtained by application of explicit and semi-implicit methods for electrons of $500 \mathrm{eV}$ and $10 \mathrm{keV}$ energy. The main conclusions are : analytical methods cannot be used below $1 \mu$; to reduce the yields of particles that cause the destabilisation of irradiated water, we need to introduce the solute at around $1 \mu s$.

\section{Introduction}

Bien que satisfaisantes pour les applications diagnostiques, les estimations dosimétriques effectuées à l'échelle macroscopique, de par les hypothèses simplificatrices qui les caractérisent (distributioñ homogène du dépôt d'énergie, contribution des électrons de basses énergies négligée...), demeurent insuffisantes quant à une meilleure interprétation des résultats cliniques associés à la radiothérapie métabolique. En effet, des calculs dosimétriques précis supposent une meilleure connaissance des paramètres physiques de base de l'irradiation au niveau cellulaire et ce, d'une part dans une perspective de radioprotection, et d'autre part dans le choix du faisceau ou du radionucléide le mieux adapté pour une situation clinique donnée.

A la lumière de ces remarques, le modèle stochastique nous a permis d'obtenir les distributions initiales et les rendements radiochimiques des espèces radiolytiques créées $10^{-12} \mathrm{~s}$ après le passage dans l'eau d'électrons d'énergies comprises entre $100 \mathrm{eV}$ et $10 \mathrm{keV}$ [19]. Ainsi et pour la première fois, le calcul des concentrations initiales des particules créées se fait sur la base de distributions réelles afin de suivre leur évolution spatio-temporelle par une méthode explicite ${ }^{*}[15]$.

L'objet de cet article est de montrer que des améliorations peuvent être apportées pour peu que l'on considère le problème sous un autre angle. En effet, l'application de la méthode semi-implicite* plus proche de la réalité physico-chimique du problème que la méthode explicite, la prise en compte d'électrons incidents d'énergie plus élevée $(10 \mathrm{keV})$ aidée par une station de travail performante, sont autant d'atouts quant à une résolution efficiente de l'équation de diffusion.

\footnotetext{
* Voir paragraphe 3.
} 
TABLEAU II

Liste des réactions chimiques considérées List of the chemical reactions considered

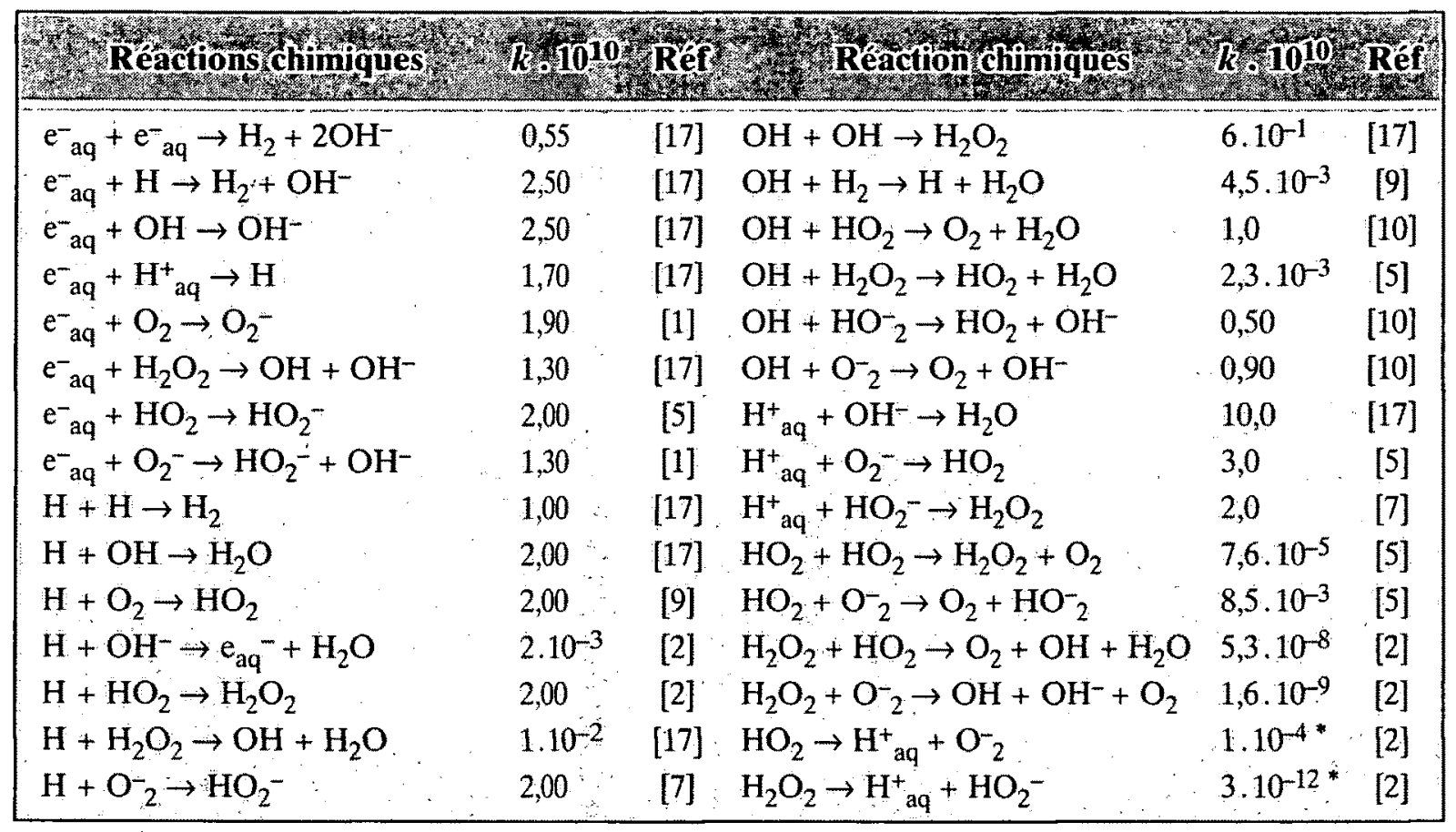

${ }^{*} k$ en s$^{-1}$.

\subsection{Distribution initiale des particules issues de la phase physico-chimique}

L'approche microscopique basée sur la méthode de Monte-Carlo permet d'obtenir la distribution initiale des particules issues de la phase physico-chimique[3].

Cependant, le modèle déterministe tel qu'il est symbolisé par l'équation (1) nécessite la donnée préalable des concentrations initiales des espèces radiolytiques à étudier. Pour ce faire, il convient de faire correspondre au domaine initial un espace composé de volumes élémentaires régulièrement répartis où chaque particule est intégrée. Un programme codé "discrétisation spatiale" permet de définir un pas spatial $\Delta x$ en fonction de l'énergie des électrons incidents, et calcule ainsi les concentrations initiales des différentes espèces à l'étude [14].

\section{Méthodes mathématiques utilisées}

\subsection{Discrétisation de l'équation de diffusion}

Pour résoudre l'équation (1), il faut la discrétiser, c'est-à-dire la remplacer par de simples équations algébriques exprimant la même information physique et pouvant être résolues numériquement. Pour cela, on transforme l'équation (1) en une équation intégrale par application de la méthode des résidus pondérés : 


$$
\iiint \int \frac{\partial C_{i}}{\partial t} w(x, y, z, t) \mathrm{d} x \mathrm{~d} y \mathrm{~d} z \mathrm{~d} t=\iiint \int\left[D_{i} \nabla^{2} C_{i}+F\right] w(x, y, z, t) \mathrm{d} x \mathrm{~d} y \mathrm{~d} z \mathrm{~d} t
$$

Si $C_{i}(x, y, z, t)$ vérifie l'équation (3) pour toute fonction test $w(x, y, z, t)$, alors les équations (1) et (3) sont équivalentes ou, en d'autres termes, elles admettent la même solution. Mais si $C_{i}((x, y, z, t)$ vérifie l'équation (3) pour un ensemble "suffisamment vaste" de fonctions $w(x, y, z, t)$ indépendantes, alors $C_{i}(x, y, z, t)$ est une solution approchée de l'équation (1) [21]. La fonction test $w(x, y, z, t)$ étant choisie égale à l'unité (méthode des éléments finis continus) [8], l'intégration de l'équation (3) entraîne des équations algébriques telles que la concentration $C_{i}$ en un point $\mathrm{P}$, centre d'un volume élémentaire à l'intérieur du domaine de calcul, est reliée à celles des points $\mathrm{E}, \mathrm{W}, \mathrm{R}, \mathrm{Q}, \mathrm{S}$, et $\mathrm{N}$ situés aux faces de ce même volume.

Cependant, notre objectif est de trouver une relation telle que $C_{i \mathrm{P}}$ soit fonction des concentrations aux points $\mathrm{E}, \mathrm{W}, \mathrm{R}, \mathrm{Q}, \mathrm{S}$, et $\mathrm{N}$, centres des volumes élémentaires avoisinants le volume considéré (Fig. 1). Pour accéder à cette formulation, nous avons adopté un schéma identique à celui des différences centres [13]. Ainsi, la méthode semi-implicite permet de remplacer l'équation (3) par l'équation algébrique :

$$
A_{\mathrm{P}} C_{i \mathrm{P}}=A_{\mathrm{E}} C_{i \mathrm{E}}+A_{\mathrm{W}} C_{i \mathrm{~W}}+A_{\mathrm{R}} C_{i \mathrm{R}}+A_{\mathrm{Q}} C_{i \mathrm{Q}}+A_{\mathrm{S}} C_{i \mathrm{~S}}^{0}+A_{\mathrm{N}} C_{i \mathrm{~N}}^{0}+K
$$

où la concentration $C_{i p}$ à l'instant $t+\Delta t$ est fonction des concentrations :

$-C_{i \mathrm{E}}, C_{i \mathrm{~W}}, C_{i \mathrm{R}}$ et $C_{i \mathrm{Q}}$ à l'instant $t+\Delta t$ (termes implicites),

- $C_{i S}^{0}$ et $X_{\text {in }}^{O}$ à l'instant $\mathrm{t}$ (termes explicites),

- $A_{\mathrm{P}}, A_{\mathrm{E}}, A_{\mathrm{W}}, A_{\mathrm{R}}, A_{\mathrm{Q}}, A_{\mathrm{S}}, A_{\mathrm{N}}$ et $K$ sont des coefficients qu'on explicitera en 3.2 .

\subsection{Calcul des coefficients relatifs à l'équation (4)}

Pour un point $\mathbf{P}$ situé à l'intérieur de la grille tridimensionnelle, l'intégration de (3) sur les intervalles suivants : $[t, t+\Delta t],[x-\Delta x / 2, x+\Delta x / 2],[y-\Delta y / 2$, $y+\Delta y / 2],[z-\Delta z / 2, z+\Delta z / 2]$ permet d'obtenir les coefficients $A_{\mathrm{P}}, A_{\mathrm{E}}, A_{\mathrm{W}}, A_{\mathrm{R}}$, $A_{\mathrm{Q}}, A_{\mathrm{S}}, A_{\mathrm{N}}$ et $K$ relatifs à l'équation (4) :

$$
\begin{gathered}
A_{\mathrm{E}}=A_{\mathrm{W}}=D_{i} \frac{\Delta x \Delta z}{\Delta y} ; A_{\mathrm{R}}=A_{\mathrm{Q}}=D_{i} \frac{\Delta y \Delta z}{\Delta x} ; A_{\mathrm{S}}=A_{\mathrm{N}}=D_{i} \frac{\Delta x \Delta y}{\Delta z} \\
A_{\mathrm{P}}=\frac{\Delta x \Delta y \Delta z}{\Delta t}+A_{\mathrm{E}}+A_{\mathrm{W}}+A_{\mathrm{R}}+A_{\mathrm{Q}}+A_{\mathrm{S}}+A_{\mathrm{N}} \\
K=\frac{\Delta x \Delta y \Delta z}{\Delta t} \cdot C_{i \mathrm{P}}^{0}+F_{\mathrm{P}}^{0} \Delta x \Delta y \Delta z
\end{gathered}
$$




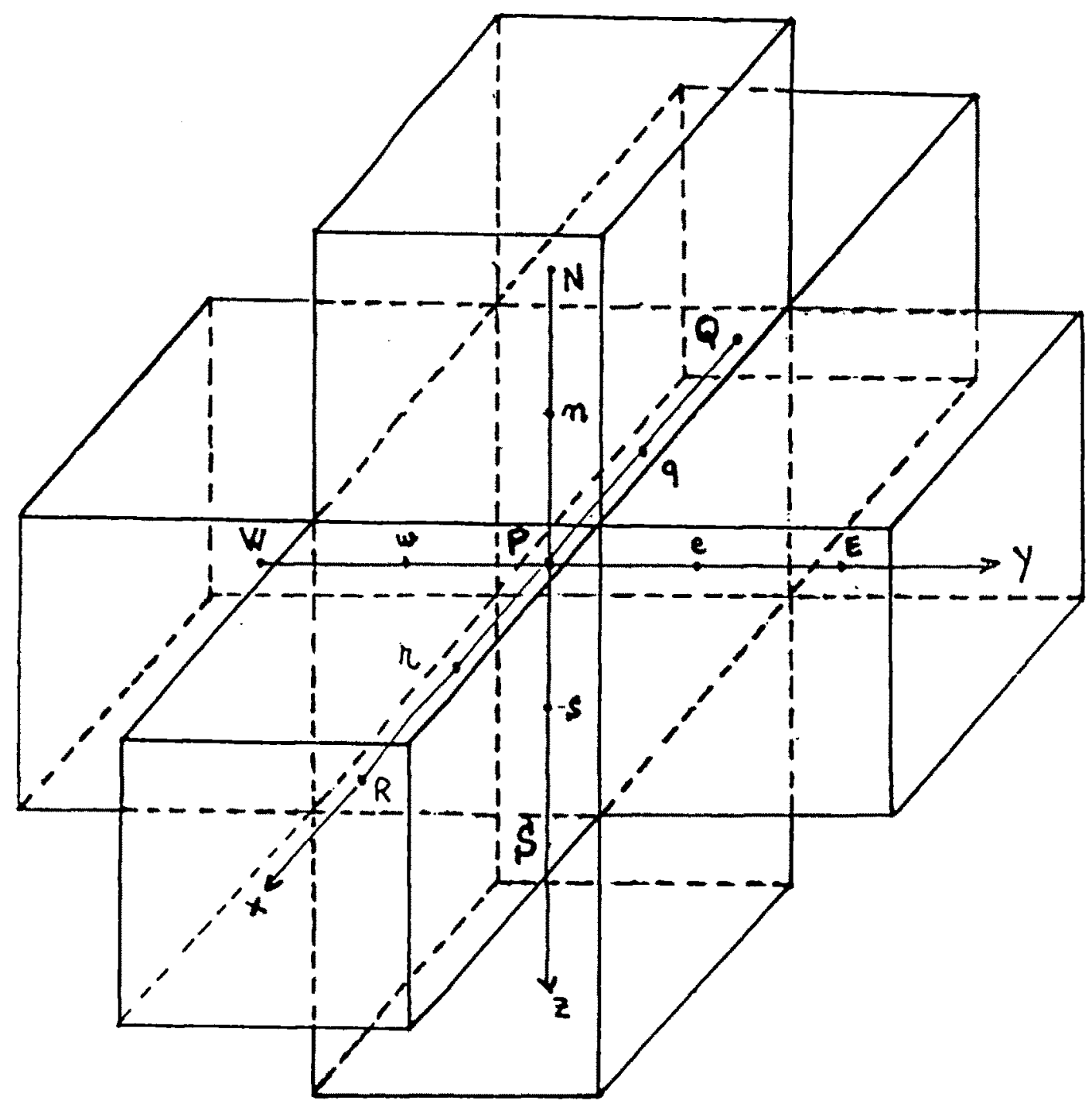

Fig. I - Voisinage immédiat d'un volume élémentaire à l'intérieur de la grille tridimensionnelle.

Immediate neighbourhood of an elementary volume located inside the 3-D net.

$C_{i p}^{0}$ est la concentration de l'espèce $i$ au point $\mathrm{P}$ à l'instant $t$,

$F_{\mathrm{P}}^{0}$ est le terme source au point $\mathrm{P}$ à l'instant $t$,

$\Delta x, \Delta y, \Delta z$ étant les pas spatiaux selon les trois directions de l'espace,

$\Delta t$ est le temps au bout duquel les variations de la concentration d'une espèce radiolytique donnée sont évaluées.

Les conditions aux limites relatives aux points extrêmes du domaine de calcul permettent de leur associer des coefficients dont la valeur varie selon la position du volume élémentaire considéré [14].

Après intégration de l'équation (3) sur tous les volumes élémentaires de l'espace des concentrations, on obtient un autre système à matrice tridiagonale par blocs, dont la résolution par la méthode de Thomas généralisée au calcul matriciel est immédiate [4]. 


\section{Mise en forme informatique}

La finesse du maillage impose un pas spatial optimal. Celui-ci dépend à la fois de l'énergie des particules incidentes et de la taille mémoire du système informatique utilisé. Pour simuler la suite des événements, nous avons adopté un pas temporel $\Delta t$ logarithmique en adéquation avec les processus de diffusion-réaction d'une part, et d'autre part avec la stabilité de la méthode de résolution. La valeur limite supérieure imposée est donnée par la relation :

$$
\Delta t=\frac{(\Delta x)^{2}}{6 . \mathrm{DIF}\left(\mathrm{H}_{\mathrm{aq}}^{+}\right)}
$$

$\Delta x$ est pris égal à $\Delta y$ et $\Delta z$, $\operatorname{DIF}\left(\mathrm{H}_{\mathrm{aq}}^{+}\right)$est la constante de diffusion de $\mathrm{H}_{\mathrm{aq}}^{+}$.

Le calcul des concentrations des espèces radiolytiques au niveau de chaque volume élémentaire pour un pas temporel $\Delta t$ est assuré par un sous-programme spécifique. Tandis que les particules diffusent, le nombre de volumes élémentaires augmente, ce qui a pour effet de bloquer nos investigations au bout d'un temps fonction de la mémoire disponible. Pour résoudre ce problème, on a fait appel à une technique de compression du domaine d'étude, entraînant ainsi la création d'un espace suffisant pour permettre de progresser dans nos calculs.

L'évolution du système est ainsi étudiée de proche en proche par injection de pas $\Delta t$ jusqu'au temps final comme le montre la figure 2 .

\section{Résultats et interprétations}

Des valeurs chiffrées de la concentration des espèces radiolytiques considérées, un ensemble de courbes d'isoconcentration sont déduites au niveau de chacun des plans du domaine irradié par des électrons d'énergies comprise entre $500 \mathrm{eV}$ et $10 \mathrm{keV}$. Cependant, devant l'abondance de matière, nous nous sommes particulièrement intéressés au devenir de l'électron solvaté et du radical hydroxyle au vu des nombreuse réactions déterminantes auxquelles ils participent (Figs. 3ad et 4ad). En effet, l'existence d'un gradient local de concentrations fait que les particules diffusent des zones d'hétérogénéité où elles ont été créées et réagissent entre elles et ce entre $10^{-12} \mathrm{~s}$ et $10^{-8} \mathrm{~s}$.

Vers $10^{-8} \mathrm{~s}$, les réactions de recombinaison sont concurrencées par les réactions entre les radicaux et les molécules produites jusqu'à l'établissement d'un équilibre thermodynamique. Celui-ci est atteint au bout d'1 $\mu$ s, et toute tentative de résolution analytique basée sur la notion de distribution à symétrie sphérique ou cylindrique. n'est possible qu'après l'écoulement de ce temps, contrairement à ce qui a été proposé $[12,17]$. 


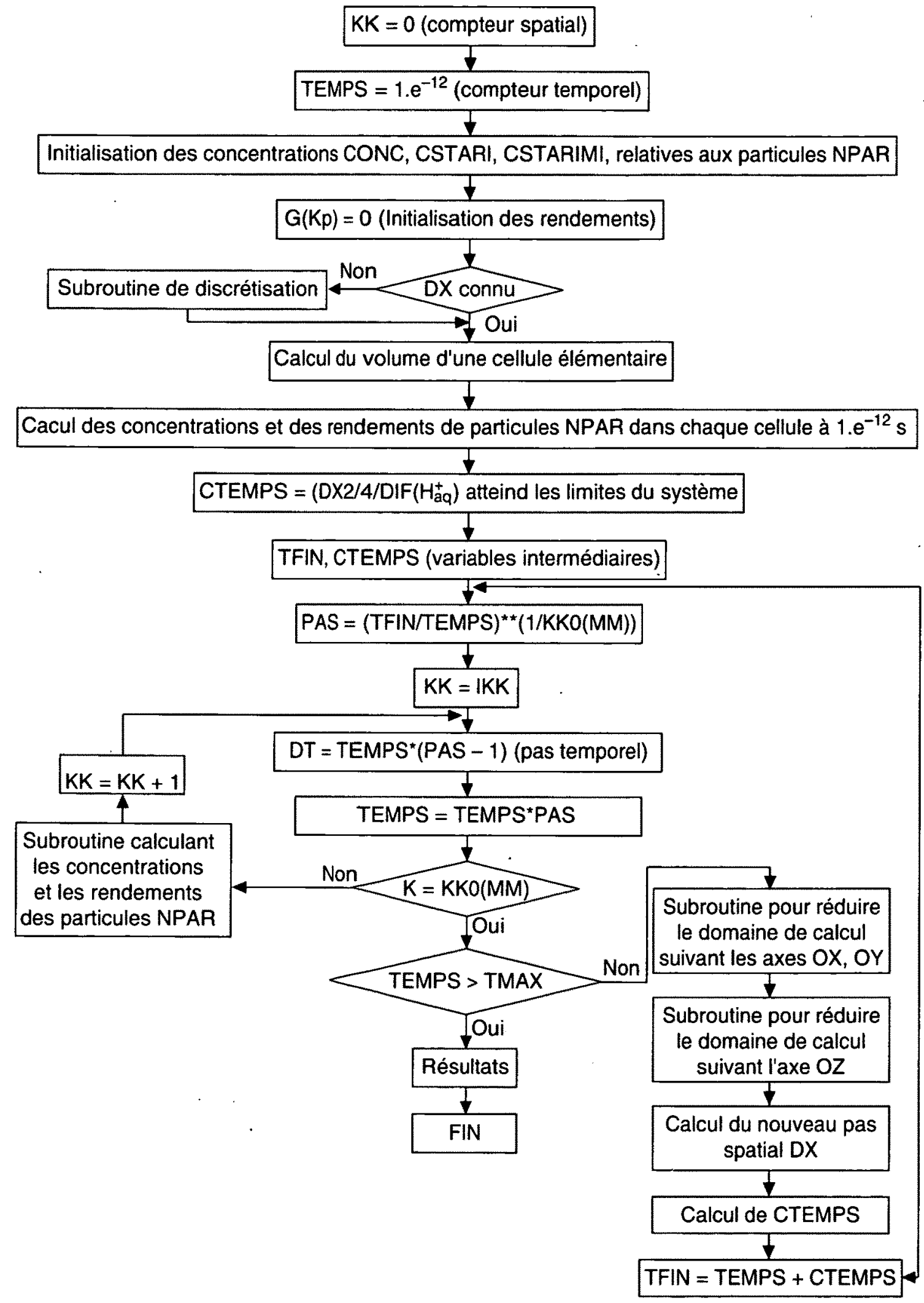

Fig. 2 - Organigramme général de calcul des concentrations et rendements radiolytiques par une méthode semi-implicite dans le cas d'un pas $\Delta t$ variable.

General diagram for concentrations and yields calculations by a semi-implicit method using a varying time step. 


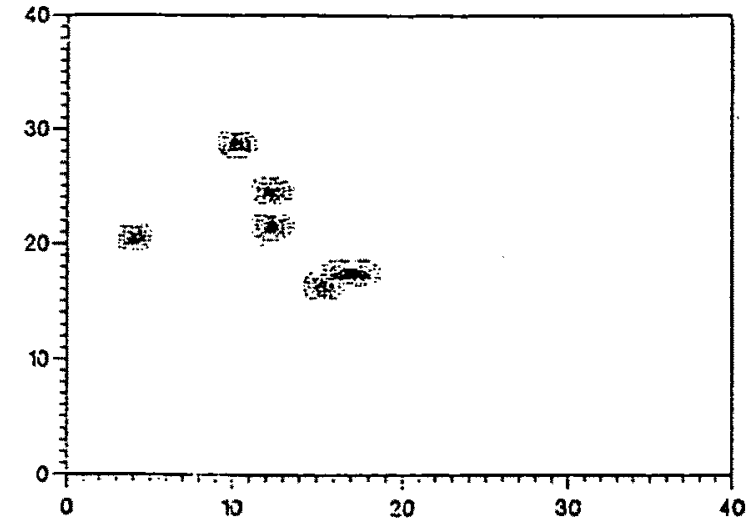

a)

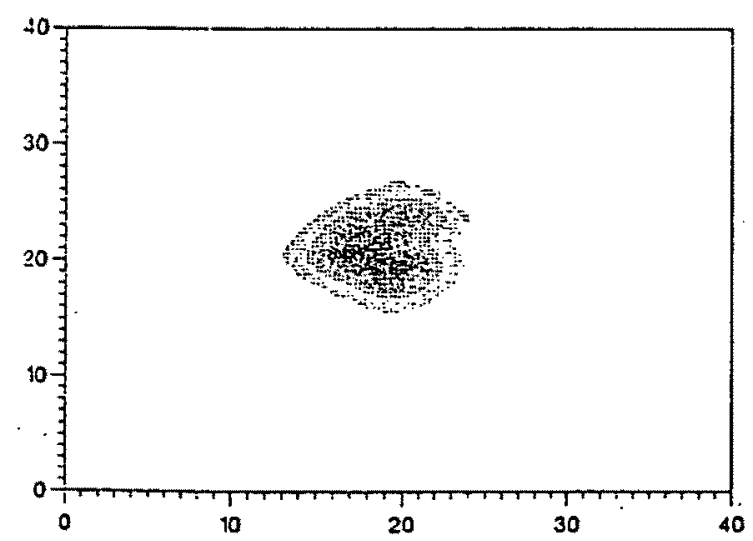

c)

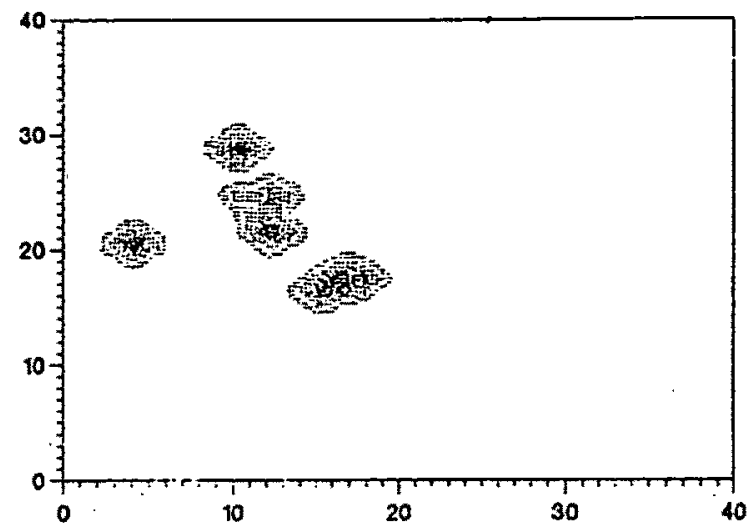

b)

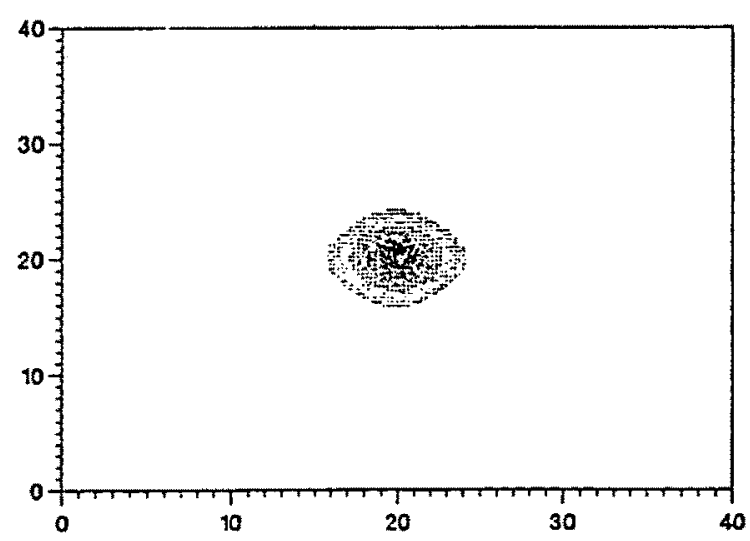

d)

Fig. 3 - Evolution spatio-temporelle des courbes d'isoconcentration de l'électron solvaté : (a) : zones d'hétérogénéités à $4.10^{-12} \mathrm{~s}$,

(b) : décroissance de la concentration de $e_{a q}^{-}$dans les zones d'hétérogénéité à $10^{-10} \mathrm{~s}$,

(c) : tendance à l'uniformité à $10^{-8} \mathrm{~s}$,

(d) : disparition des zones d'hétérogénéité à $10^{-6} \mathrm{~s}$.

Spatio-temporal evolution of iso-concentration curves of solvated electrons :

(a): heterogeneous areas at $4.10^{-12} \mathrm{~s}$,

(b): solvated electron concentration decrease in the heterogeneous areas at $10^{-10} \mathrm{~s}$

(c) : $e_{a q}^{-}$concentrations become little uniform at $10^{-8} s$,

(d) : disappearance of the heterogeneous areas at $10^{-6} \mathrm{~s}$.

En outre, le rendement radiochimique, bien que moins puissant que la notion de concentration, a été utilisé pour rendre compte de manière globale du devenir des espèces radiolytiques présentes à $10^{-12} \mathrm{~s}\left(\mathrm{e}^{-} \mathrm{aq}, \mathrm{H}, \mathrm{OH}, \mathrm{H}^{+}{ }_{\mathrm{aq}}\right.$, $\left.\mathrm{H}_{2}, \mathrm{H}_{2} \mathrm{O}_{2}\right)$ et celles créées au cours du temps $\left(\mathrm{O}_{2}, \mathrm{O}^{-}, \mathrm{OH}^{-}, \mathrm{HO}_{2}, \mathrm{HO}_{2}^{-}\right)$. Ainsi, pour une particule du type $X$, le rendement radiochimique $G(X)$ est donné par la relation :

$$
G(X)=100 / N(X) / E
$$

$N(X)$ est nombre de particules de type $X$ dans la distribution à l'instant $t$, $E$ est l'énergie déposée par l'électron incident, exprimée en $\mathrm{eV}$. 
A. SAIFI

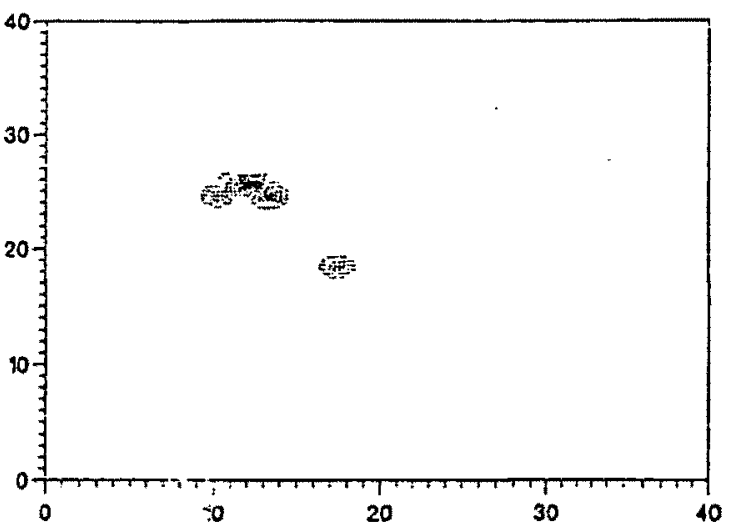

a)

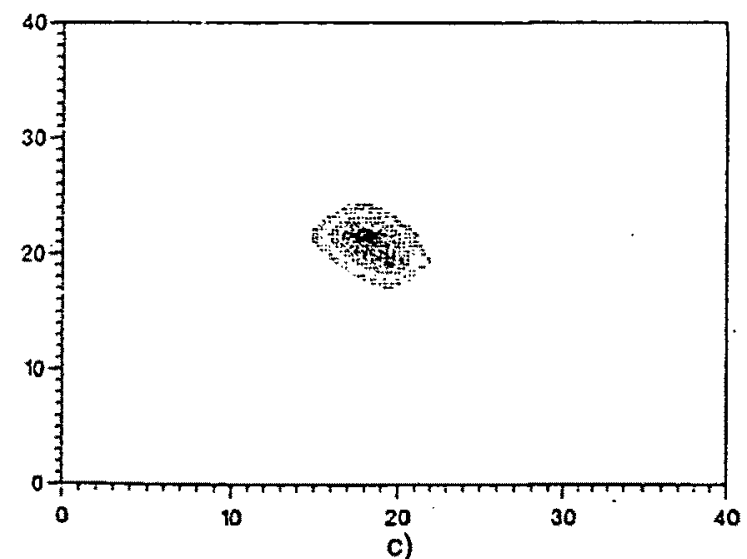

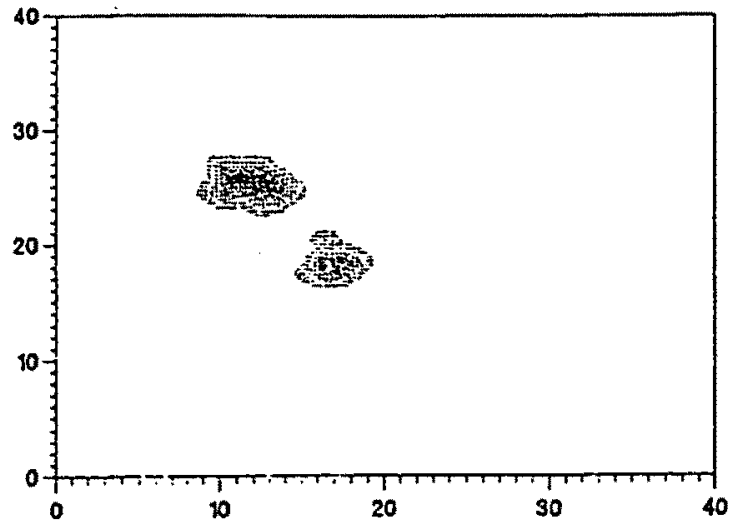

b)

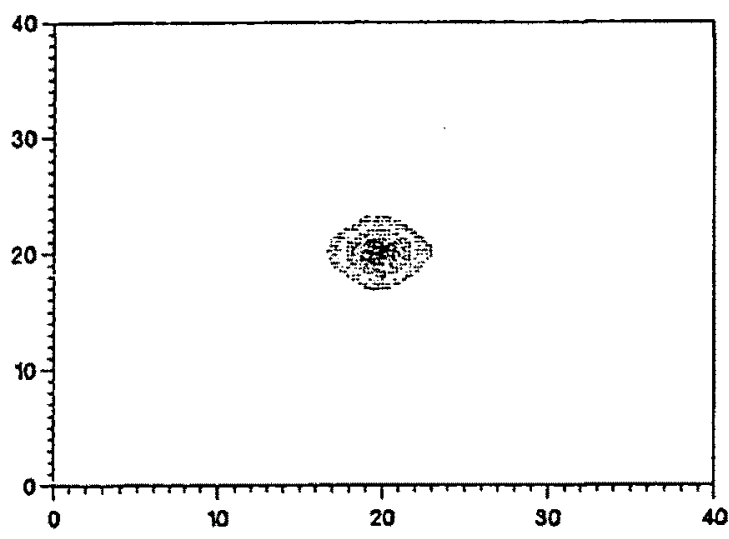

d)

Fig. 4 - Evolution spatio-temporelle des courbes d'isoconcentration du radical hydroxyle (a) : zones d'hétérogénéités à $4.10^{-12} \mathrm{~s}$,

(b) : décroissance de concentration de $\mathrm{OH}$ dans les zones d'hétérogénéité à $10^{-10} \mathrm{~s}$,

(c) : tendance à l'uniformité à $10^{-8}$ s,

(d) : disparition des zones d'hétérogénéité à $10^{-6} \mathrm{~s}$.

Spatio-temporal evolution of iso-concentration curves of hydroxyl radical : (a): heterogeneous areas at $4.10^{-12} \mathrm{~s}$,

(b): OH concentration decrease in the heterogeneous areas at $10^{-10} \mathrm{~s}$,

(c) : OH concentrations become little uniform at $10^{-8} \mathrm{~s}$,

(d): disappearance of the heterogeneous areas at $10^{-6} s$.

Les figures $5 \mathrm{ab}$ et $6 \mathrm{ab}$ montrent l'évolution des rendements radiochimiques au cours du temps des particules étudiées pour respectivement des énergies déposées égales à $500 \mathrm{eV}$ et $10 \mathrm{keV}$. On remarque d'une part la confirmation des résultats obtenus par le biais de la notion de concentration (recombinaisons, réactions entre radicaux), d'autre part plus l'énergie déposée est élevée plus la distribution initiale des particules est importante, donc le nombre des réactions est plus conséquent. Ce qui explique probablement la disparition (l'apparition) plus rapide des espèces radiolytiques dans le cas d'une énergie de $10 \mathrm{keV}$ par rapport à $500 \mathrm{eV}$.

La figure 7ab permet une comparaison entre les rendements radiochimiques obtenus par les méthodes explicite et semi-implicite pour des énergies déposées de $500 \mathrm{eV}$ et $10 \mathrm{keV}$. 

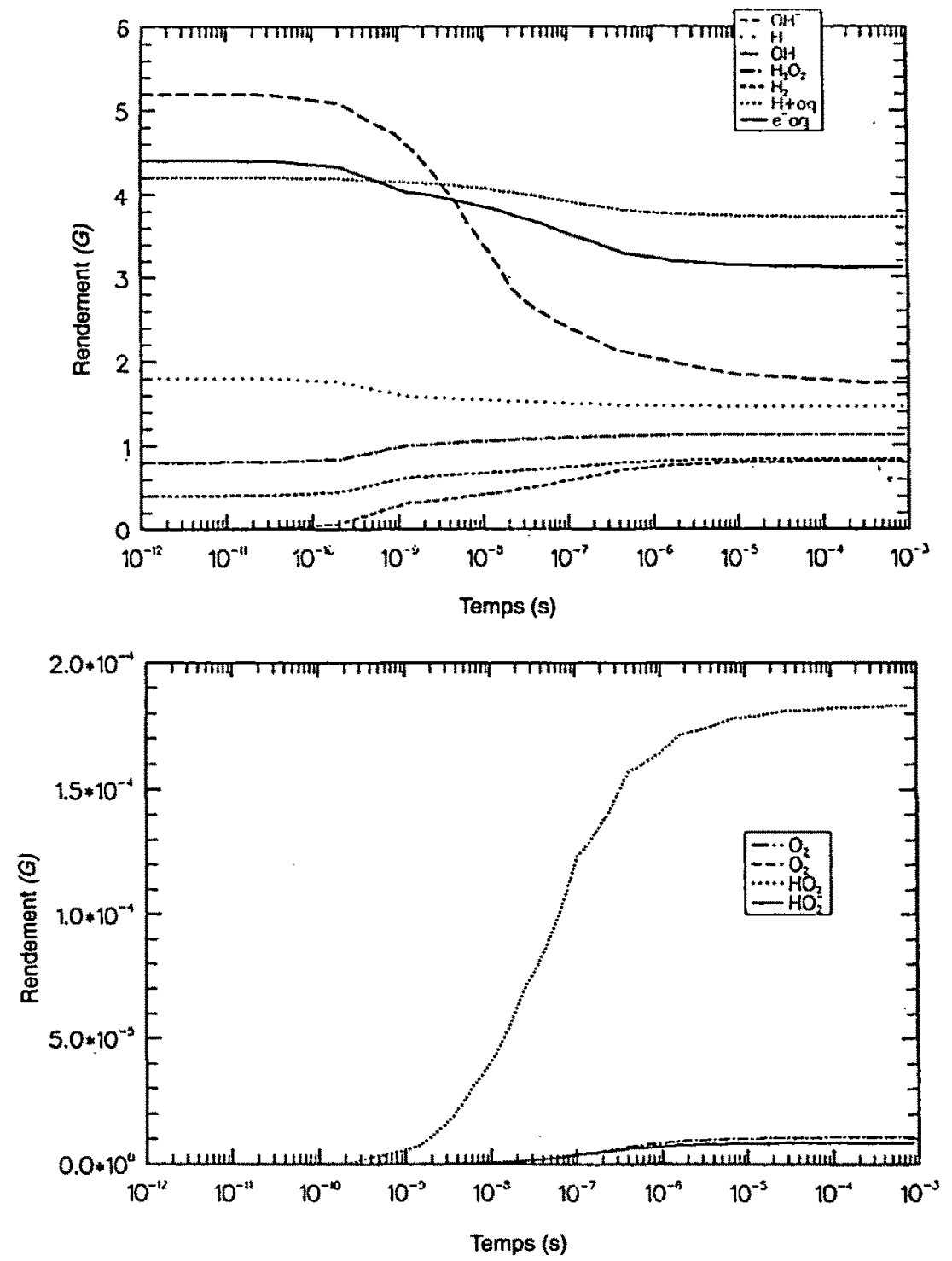

Fig. 5 - Evolution temporelle des rendements radiochimiques pour une énergie de $500 \mathrm{eV}$ : (a) : principales espèces radicalaires, (b) : particules à faible concentration.

Temporal evolution of yields for an energy of $500 \mathrm{eV}$ :

(a): main radiolytic species, (b) : low concentration species.

En effet, les réactions et diffusions ignorées par la méthode explicite durant l'intervalle de temps $\Delta t$, sont prises en compte par la méthode semi-implicite, en ce sens que la concentration $C_{i \mathrm{p}}$ dépend dans une large mesure de l'état d'évolution des concentrations aux points voisins du maillage. Cela se traduit par une décroissance (croissance) plus accentuée au niveau des courbes de rendement d'autant plus que l'énergie est plus grande. 

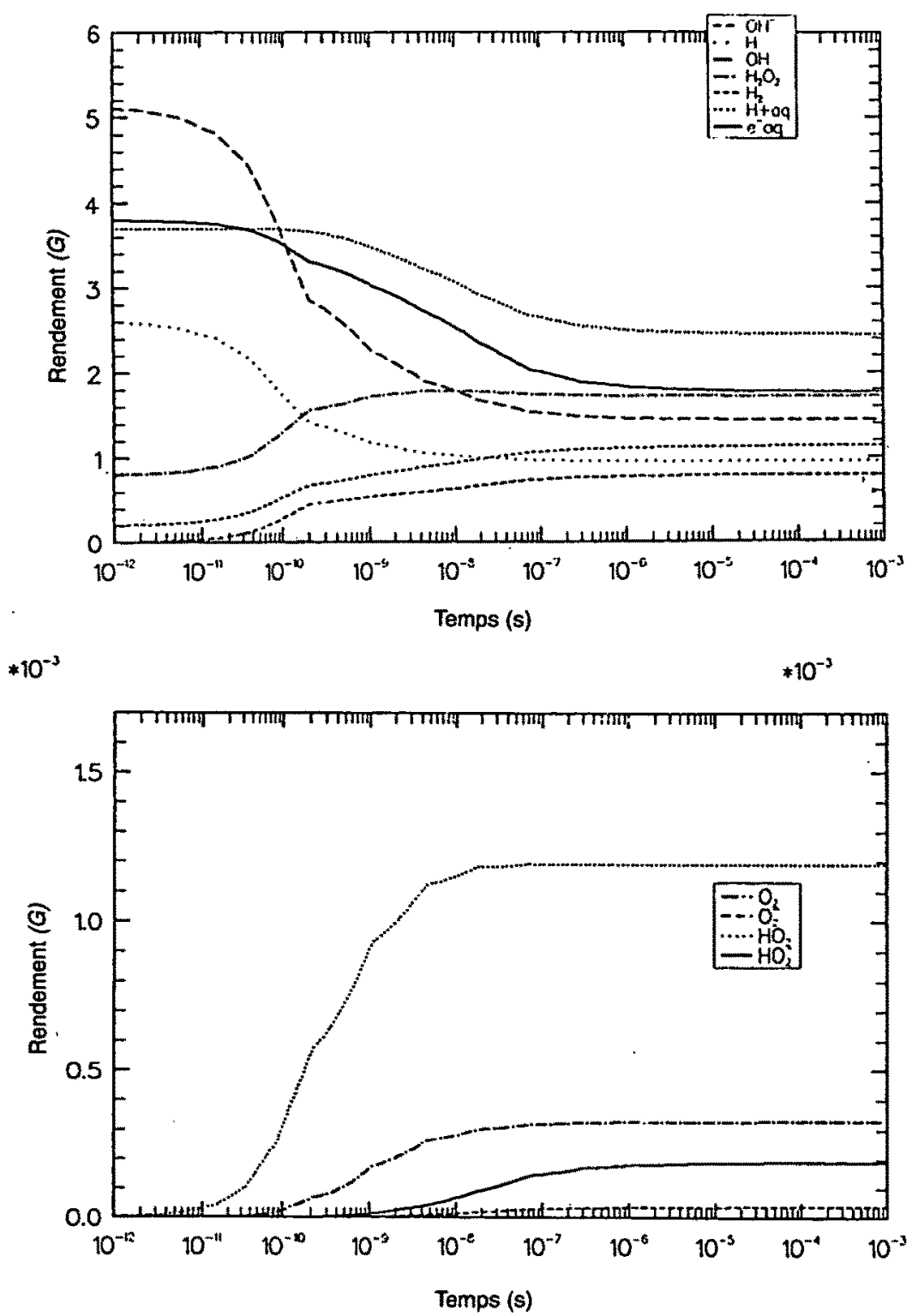

Fig. 6 - Evolution temporelle des rendements radiochimiques pour une énergie de $10 \mathrm{keV}$ : (a) : principales espèces radicalaires, (b) : particules à faible concentration

Temporal evolution of yields for an energy of $10 \mathrm{keV}$ :

(a) : main radiolytic species, (b) : low concentration species.

\section{Comparaisons avec les autres modèles}

La figure 8a montre les points expérimentaux de Jonah entre 100 et $30 \mathrm{~ns}$ [11], le point expérimental de Sumiyoshi à 30 ps [18], et les variations de $G\left(\mathrm{e}^{-}\right.$aq $)$obtenus par le modèle stochastique [3] que nous comparons aux variations du rendement radiochimique de l'électron solvaté obtenu par notre modèle. Une comparaison des variations relatives de ces rendements entre $10^{3}$ ps et 24 ns normalisées à 1 ns est présentée en figure $8 \mathrm{~b}$. Une trace de 10 $\mathrm{keV}$ a été utilisée pour comparer nos résultats avec ceux d'autres modèles 

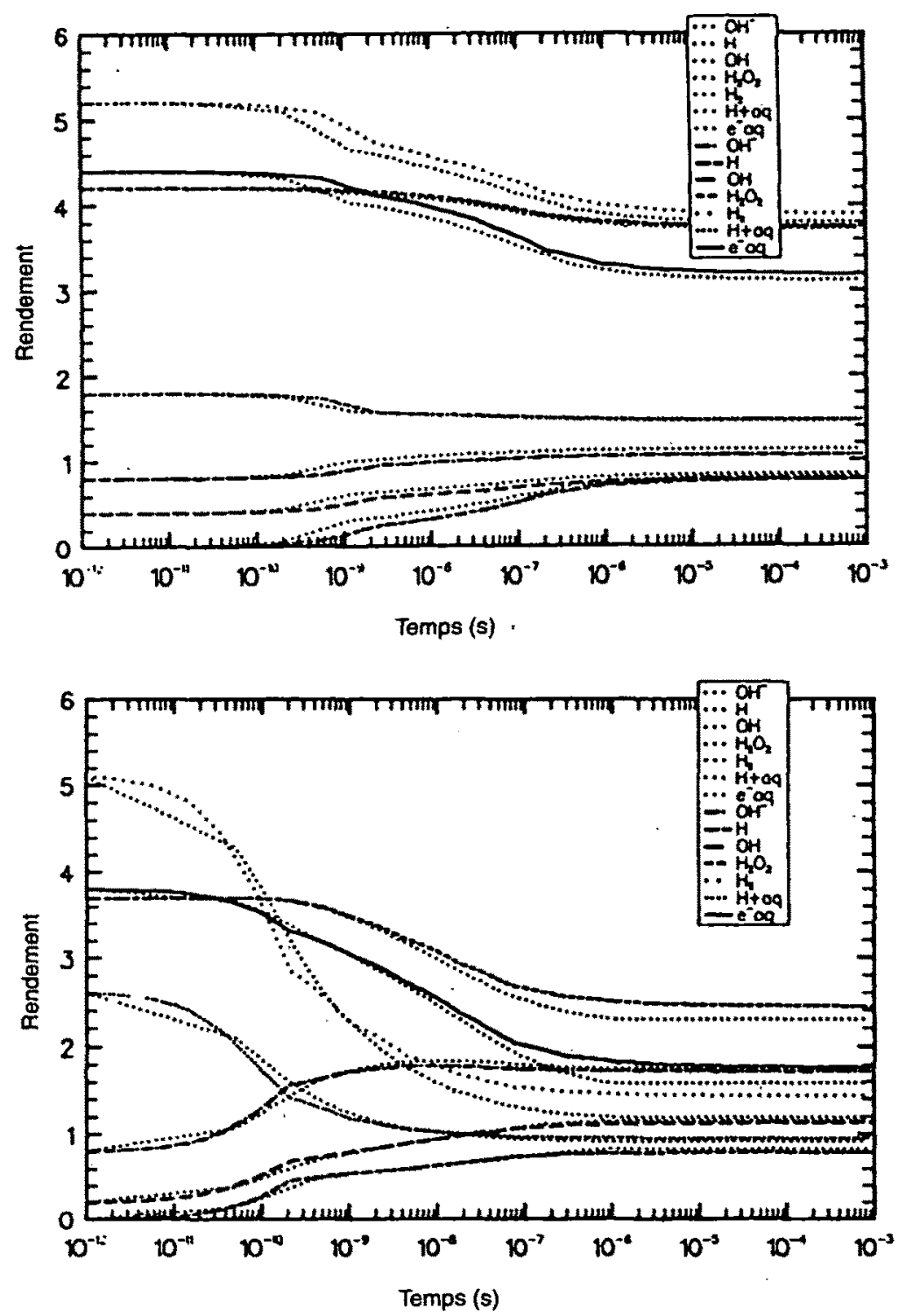

Fig. 7 - Comparaison des rendements radiochimiques obtenus par les méthodes explicite et semi-implicite: (a) : pour une énergie de $500 \mathrm{eV},(b)$ : pour une énergie de $10 \mathrm{keV}$.

Comparison of yields obtained by the explicit and semi-implicit methods : (a) : for an energy of $500 \mathrm{eV}$, (b) : for an energy of $10 \mathrm{keV}$.

théoriques tels que ceux de Trumbore [20], Schwarz [17] et Baudré [3]. Malgré l'écart entre les énergies mises en jeu, les variations du rendement de l'électron hydraté des différents modèles sont comparables (Fig. 9a). Ceci est d'ailleurs comfirmé par la figure $9 \mathrm{~b}$, où les variations relatives du radical hydroxyle $\mathrm{OH}$ normalisées à 0,2 ns sont présentées. 

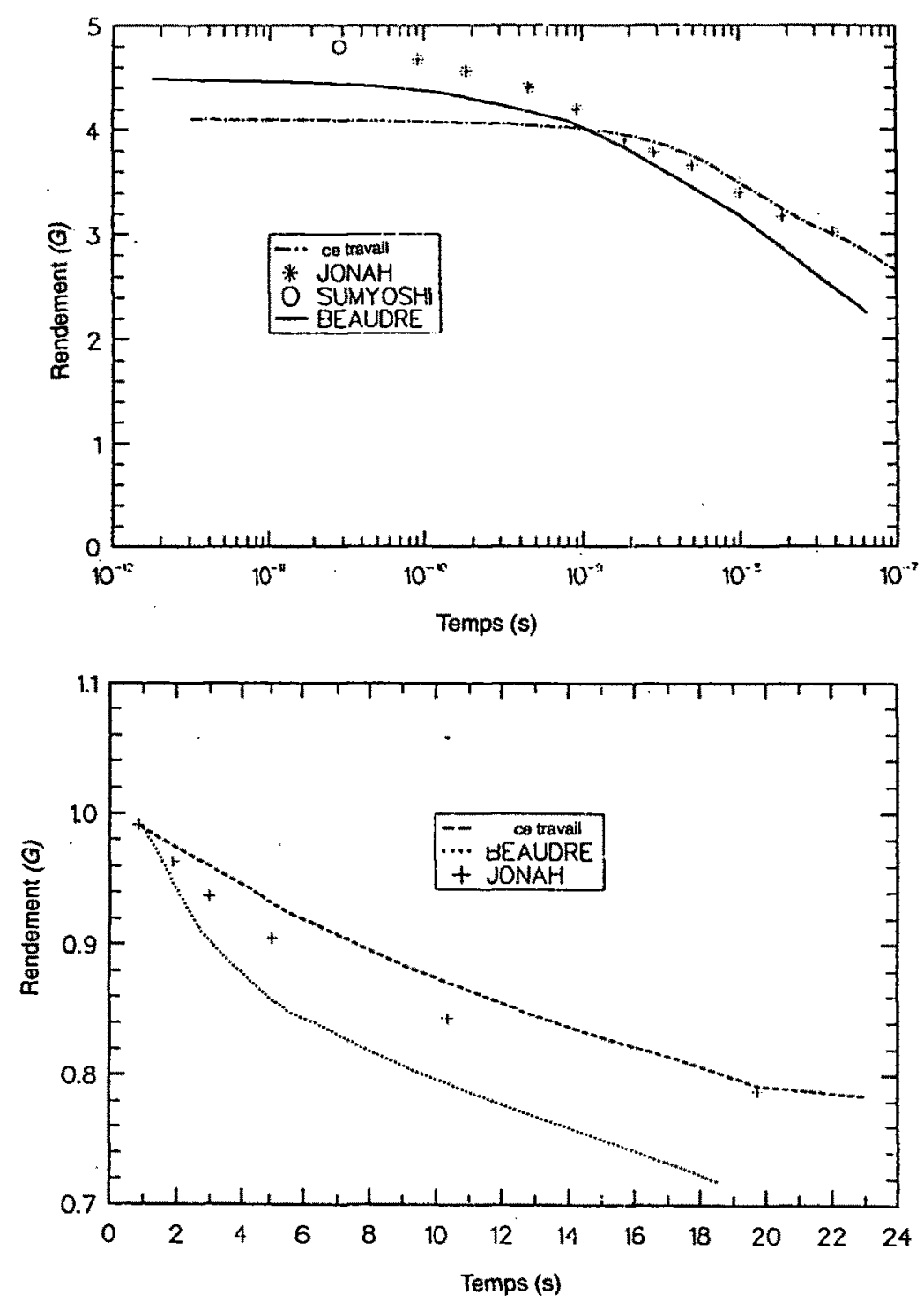

Fig. 8 - Variation de $G\left(e_{a y}^{-}\right)$au cours du temps $(10 \mathrm{keV}):(a)$ : modèle stochastique [3], points expérimentaux de Jonah [11] et point expérimental de Sumyoshi [18], (b) : valeurs normalisées à $1 \mathrm{~ns}$.

Variation with time of $G\left(e_{a q}^{-}\right)(10 \mathrm{keV}):(a):$ stochastic model [3], experiments of Jonah [11] and Sumyoshi [18], (b) : values normalised at $1 \mathrm{~ns}$.

\section{Conclusion et perspectives}

Notre contribution à l'étude de la phase chimique se distingue de celles menées soit analytiquement par Samuel [16] et Mozumder [12], soit numériquement par Schwarz [17], Burns [6] et Trumbore [20], en ce sens qu'elle utilise une technique numérique telle que celle employée jusque là par Patankar [13] dans le domaine du transfert de la chaleur. En outre, la consistance des 
RESOLUTION DE L'ÉQUATION DE DIFFUSION PAR UNE METHODE SEMI-IMPLICITE
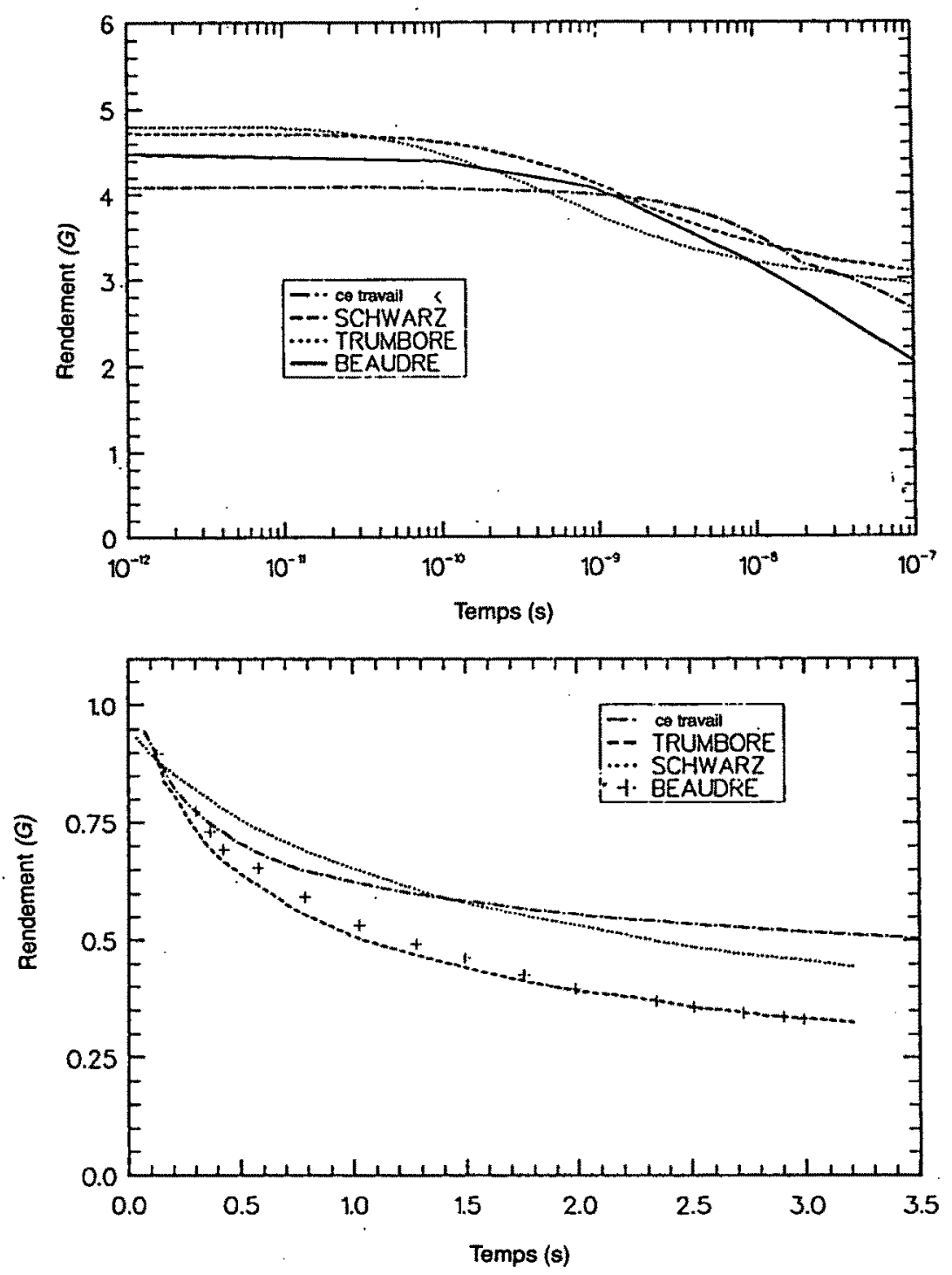

Fig. 9 - Variation de $G\left(e_{a y}^{-}\right)$et de $G(\mathrm{OH})$ au cours du temps $(10 \mathrm{keV})$ : (a) : modèle stochastique [3], Schwarz [17] et Trumbore [20], (b) : rendements de G (OH) normalisés à 0,2 ns.

Variation with time of $G\left(e_{a x}^{-}\right)$and $G(O H)(10 \mathrm{keV}):(a):$ stochastic model [3], Schwarz [17] and Trumbore [20], (b) : yields of $G(O H)$ normalised at 0,2 ns.

codes élaborés se caractérise d'une part par le nombre de réactions chimiques considérées, d'autre part par son pouvoir d'adaptation aux différentes méthodes numériques.

Les résultats obtenus sont dans une certaine mesure comparables à ceux d'autres modèles, qu'ils soient théoriques ou expérimentaux, malgré les différentes énergies utilisées par les uns et les autres. L'application de la méthode semi-implicite du type Crank-Nicholson nous a permis, de par les résultats obtenus, de constater : 
- l'inefficience des méthodes analytiques avant l'écoulement d'un temps de l'ordre de la microseconde (Figs. 3 et 4);

- que la prise en compte d'un soluté quelconque n'est nécessaire qu'autour de la microseconde pour réduire les rendements des particules à l'origine du déséquilibre du milieu irradié (Fig. 5).

Enfin, après avoir maîtriser les grandes lignes de notre problème, nous comptons avec l'avènement de l'outil informatique, non seulement tenir compte d'un nombre plus important de réactions chimiques, mais aussi adopter comme technique de résolution la méthode implicite. Celle-ci demeure la plus indiquée quant à la description de l'évolution des espèces radiolytiques, en ce sens que tous les termes composant lé. second membre de l'équation (4) sont pris à l'instant $t+\Delta t$.

Remerciements. J'exprime ma grande reconnaissance et mes remerciements à $M$. Terrisol et D. Blanc, directeur du centre de physique atomique de l'Université Paul Sabatier de Toulouse, pour leur disponibilité et leur dévouement.

\section{RÉFÉRENCES}

[1] ANBAR M., BAMBENEK M., ROSS A.B. - Selected specific rates of reactions of transients from water in aqueous solutions. I. Hydrated electron. Report NSRDSNBS43. Washington, DC : National bureau of standards, 1973.

[2] ANBAR M. FARHATAZIZ, ROSS A.B. - Selected specific rates of reactions of transients from water. II. Hydrogen atom. Report NSRDS-NBS41. Washington, DC : National bureau of standards, 1975.

[3] BEAUDRÉ A. - Simulation spatio-temporelle sur ordinateur de processus radiolytiques induits dans l'eau par des électrons. Thèse de doctorat d'Université, $\mathrm{n}^{\circ} 371$, Toulouse, 1988.

[4] BOUMAHRAT M., GOURDIN A. - Méthodes numériques appliquées. Alger : OPU, 1983.

[5] BURNS W.G., MAY R., BAVERSTOCK K.F. - Oxygen as a product of water radiolysis in high LET tracks. I. The origin of the hydroperoxyl radical in water radiolysis. Radiat. Res., 1981, 86, 1-19.

[6] BURNS W.G., SIMS H.E., GOODALL J.A.B. - Radiation chemical diffusion kinetic calculation with prescribed and non prescribed diffusion. Radiat. Phys. Chem., $1984,23,143-150$.

[7] BOYD A.W., CARVER M.B., DIXON R.S. - Computer and experimental product concentrations in the radiolysis water. Radiat. Phys. Chem., 1980, 5, 177-185.

[8] CHATWITI A. - Etude de l'évolution temporelle des électrons dans un gaz faiblement ionisé. Thèse de doctorat d'Etat, $\mathrm{n}^{\circ} 1381$, Toulouse, 1988. 
[9] DORFMAN L.M., MATHESON M.S. - Pulse radiolysis. Prog. React. Kin., 1965, 3, 237-301.

[10] FARHATAZIZ, ROSS A.B. - Selected specific rates of reactions of transients from water aqueous solutions. Hydroxyl radical and perhydroxyl radical and their radical ion. Report NSRDS-NBS59. Washington, DC : National bureau of standards, 1977.

[11] JONAH C.D., HART E.J., MATHESON M.S. - Yield and decay of the hydrated electron at times greater than 200 ps. J. Phys. Chem., 1973, 77(15), 1838-1843.

[12] MOZUMDER A., MAGEE J.L. - A simplified approach to diffusion controlled radical reactions in the tracks of ionizing radiations. Radiat. Res., 1966, 28, 215-231.

[13] PATANKAR S.V. - Numerical heat transfer and fluid flow. Paris : McGraw-Hill, 1983.

[14] SAIFI A. - Application de l'approche déterministe à l'étude sur ordinateur des processus radiolytiques induits dans l'eau par des électrons. Thèse de doctorat d'Université, n 915, Toulouse, 1991.

[15] SAIFI A., TERRISSOL M., ROCH M. - Application de l'approche déterministe à l'étude sur ordinateur des processus radiolytiques induits dans l'eau par des électrons. $30^{\mathrm{e}}$ Congrès de la Société française des physiciens d'hôpital, Rennes, 6-8 juin, 1991, 305-316.

[16] SAMUEL A.H., MAGEE J.L. - Theory of radiation chemistry. II. Effects track in radiolysis of water. J. Chem. Phys., 1953, 21(6), 1080-1087.

[17] SCHWARZ H.A. - Application of the spur diffusion model to the reaction chemistry of aqueous solutions. J. Chem. Phys., 1969, 73(6), 1928-1937.

[18] SUMIYOSHI T., KATAYAMA M. - The yield of hydrated electron at 30 ps. Chem. Lett., 1982, 1887-1890.

[19] TERRISSOL M., BEAUDRÉ A. - Simulation of space and time evolution of radiolytic species induced by electrons in water. Radiat. Prot. Dosim., 1990, 31, 171-175.

[20] TRUMBORE C.N., SHORT D.R., FANNING J.E., OLSON J.H. - Effect of pulse dose on hydrated electron decay kinetics in the pulse radiolysis of water, a computer modeling study. J. Chem. Phys., 1978, 82(26), 2762-2767.

[21] ZIENKIEWICZ O.C. - La méthode des éléments finis. Paris : McGraw-Hill, 1971. 